\title{
Three-form multiplet and inflation
}

\section{Emilian Dudas}

CPhT, Ecole Polytechnique,

91128 Palaiseau Cedex, France

Deutsches Elektronen-Synchrotron DESY, 22607 Hamburg, Germany

E-mail: emilian.dudas@cpht.polytechnique.fr

ABSTRACT: Most successful models of inflation in supergravity have a shift symmetry for the inflaton and contain a stabilizer field coupled to the inflaton in a particular way. We argue that the natural interpretation of the stabilizer, from the viewpoint of the shift symmetry, is a three-form multiplet. Its coupling to the inflaton is uniquely determined by the shift symmetry and the invariance under three-form gauge transformations and has a natural string theory interpretation.

KEYWORDs: Supergravity Models, Supersymmetric Effective Theories

ARXIV EPRINT: 1407.5688 


\section{Contents}

1 Introduction and conclusions 1

2 Three-form and shift symmetry 3

2.1 Dual formulation 4

3 Three-form multiplet in supersymmetry and stabilizer multiplet in inflation

3.1 Dual formulation

3.2 Corrections to the inflaton potential 7

4 Supergravity formulation of the three-form multiplet $\quad 8$

4.1 Chaotic inflation 8

4.2 The Starobinsky model 9

5 Embedding in string theory $\quad 11$

\section{Introduction and conclusions}

Inflation [1-3] is an attractive scenario for explaining the initial conditions of the early universe. An exponential phase of the expansion of the universe is generated by a scalar field $\varphi$, the inflaton, with a small mass (compared to the Planck mass) $\mu$. The smallness of the inflaton mass suggests a pseudo-Nambu-Goldstone origin. Probably the best option proposed in the literature is a global shift symmetry $\varphi \rightarrow \varphi+c$, eventually broken to a discrete subgroup [4-9].

For trans-Planckian field values the contributions of Planck-scale suppressed higherdimensional operators to the inflationary potential are generically relevant. It is therefore important to consider large-field inflation in the context of some ultraviolet completion, for which string theory is the leading candidate, described by supergravity in its low-energy limit. In supergravity, the usual $\eta$-problem can be avoided for a shift symmetric Kähler potential [10] $\left.K=K(\phi+\bar{\phi})^{2}\right)$. The invariance is here with respect to $\phi \rightarrow \phi+i c$, where $c$ is a real constant, and the inflaton is $\varphi=\sqrt{2} \operatorname{Im} \phi$.

One of the simplest realization of inflation is of large-field, type, achieved with a free massive scalar field, $V=\mu^{2} \varphi^{2}$. In addition to primordial curvature perturbations, which have been measured with remarkable accuracy [11], it predicts sizeable tensor perturbations [12] for which evidence has been reported recently [13].

However, the simplest supersymmetric extension of the potential $\mu^{2} \varphi^{2}$ defined by the superpotential

$$
W=\frac{1}{2} \mu \phi^{2}
$$


has a well-known problem generated by the shift symmetry. Due to the negative term $-3|W|^{2}$ in the supergravity scalar potential, for large values of the inflaton field the potential becomes $V(\varphi) \sim-3 \mu^{2} \varphi^{4}$ and the potential is unbounded from below.

The problem can be avoided by introducing an additional 'stabilizer field' $S$, which has no shift symmetry in the Kähler potential [10], i.e.,

$$
K=K\left((\phi+\bar{\phi})^{2},|S|^{2}\right)
$$

together with the superpotential

$$
W_{\mathrm{inf}}=\mu S \phi
$$

which breaks the shift symmetry softly. This model has been generalized to a class of chaotic inflation models by replacing the inflaton field $\phi$ by a function $f(\phi)$ in the superpotential $[14,15]$. For recent studies of chaotic inflation in supergravity and string theory, see [16-25] and [26-33], respectively.

Another popular inflationary model is the Starobinsky model [34], which has a dual interpretation. On one hand, it is a gravitational theory with a higher-derivative term $R^{2}$. On the other hand, it can be described as Einstein gravity coupled to a scalar, with a very particular scalar potential. The model was generalized to supergravity in [35-37], where it was shown that, in a chiral multiplet formulation, in addition to the inflaton multiplet, there is a second chiral multiplet needed. ${ }^{1}$ It was subsequently shown in [39] that the stabilizer needs additional interactions in order to stabilize its vev to zero during inflation. In the chiral formulation, the second chiral multiplet can also be replaced by a nonlinear superfield, where the corresponding scalar is absent [40]. The couplings of this second chiral multiplet to the inflaton are very similar to the previously discussed case of the stabilizer field in chaotic inflation and could plausibly have a similar microscopic origin.

One of the open questions is the origin of the shift-symmetry breaking in (1.3). It seems unnatural from a string theory viewpoint to mix a field with a shift-symmetry to another field with no such symmetry. This is true in particular in flux compactifications, which is a generic framework invoked for generating such superpotential mass terms. In the following we propose a natural interpretation of the stabilizer field and of such a coupling in terms of a three-form multiplet, both from the viewpoint of the soft breaking of the shift symmetry and from string theory. More precisely, we will show that the mass term (1.3) is uniquely singled out by requiring the shift symmetry $\phi \rightarrow \phi+i c$ and invariance under the three-form gauge symmetry.

The three-form was to our knowledge used in chaotic inflaton for the first time in [7-9], which noticed the nice role of the shift-symmetry in this case, interpreted it as a "natural inflation" setup. It was also discussed recently in $[32,33]$ in a string theory setup, as a concrete F-term string realization of axion-monodromy [5, 6, 41-43].

\footnotetext{
${ }^{1}$ It is also possible to realize the Starobinsky model by using massive vector multiplets [38].
} 


\section{Three-form and shift symmetry}

Let us start from a lagrangian containing a scalar $\varphi$ and a three-form field $C_{m n p}$, having a global shift symmetry $\varphi \rightarrow \varphi+c$, up to boundary terms,

$$
\mathcal{S}_{0}=\int d^{4} x\left\{-\frac{1}{2}(\partial \varphi)^{2}-\frac{1}{2 \times 4 !} F_{m n p q}^{2}+\frac{\mu}{24} \varphi \epsilon^{m n p q} F_{m n p q}\right\},
$$

where

$$
F_{m n p q}=\partial_{m} C_{n p q}+3 \text { perm. . }
$$

For future convenience we define

$$
F=\frac{1}{4 !} \epsilon^{m n p q} F_{m n p q}, F_{m n p q}=-\epsilon^{m n p q} F .
$$

The lagrangian (2.1) has actually to be supplemented with a boundary term

$$
\mathcal{S}_{b}=\frac{1}{6} \int d^{4} x \partial_{m}\left(F^{m n p q} C_{n p q}-\mu \varphi \epsilon^{m n p q} C_{n p q}\right),
$$

in order to find the correct field equations. It is interesting to notice that, whereas the "bulk" action (2.1) has a shift symmetry $\varphi \rightarrow \varphi+c$ only up to boundary terms, the total action

$$
\begin{array}{r}
\mathcal{S}=\mathcal{S}_{0}+\mathcal{S}_{b}=\int d^{4} x\left\{-\frac{1}{2}(\partial \varphi)^{2}-\frac{1}{2 \times 4 !} F_{m n p q}^{2}-\frac{\mu}{6} \epsilon^{m n p q} \partial_{m} \varphi C_{n p q}\right\} \\
+\frac{1}{6} \int d^{4} x \partial_{m}\left(F^{m n p q} C_{n p q}\right)
\end{array}
$$

is exactly shift symmetric. A massless three-form field has no on-shell degrees of freedom. As such, it can be integrated out via its field eqs.

$$
\partial^{m} F_{m n p q}=+\mu \epsilon_{m n p q} \partial^{m} \varphi
$$

whose solution is given by

$$
F=+\mu \varphi-f_{0},
$$

where $f_{0}$ is a constant, which is to be interpreted as a flux. It was argued in [44] that $f_{0}$ is quantized in units of the fundamental electric coupling $f_{0}=n e^{2}$, fact that was argued to have important consequences for the landscape of string theory. After doing so, the final lagrangian takes the form

$$
\mathcal{S}=\int d^{4} x\left\{-\frac{1}{2}(\partial \varphi)^{2}-\frac{1}{2}\left(\mu \varphi-f_{0}\right)^{2}\right\} .
$$

Notice that the boundary term $\mathcal{S}_{b}$ is crucial in obtaining the correct action. Ignoring it leads to the wrong sign of the last term in (2.8), fact that created confusion in the past. In the form (2.8), it is clear that the theory describes a massive scalar field of mass $\mathrm{m}$, whereas the flux $f_{0}$ determines the ground state. It is remarkable that, whereas the action has a shift symmetry that would naively suggests that the scalar is massless, actually the 
field acquires a topological mass [7-9]. In the final formulation (2.8) the shift symmetry seems completely broken. There is one sense in which a discrete subgroup of it is preserved, however, namely

$$
\varphi \rightarrow \varphi+\frac{e^{2}}{\mu} \quad, \quad n \rightarrow n+1,
$$

where $n$ is the flux quantum. A nice string intepretation of (2.9) in terms of axion monodromy and brane nucleation was recently provided in [32, 33], following [7-9].

\subsection{Dual formulation}

The dual formulation contains only a massive three-form field. The duality proceeds starting from the master action

$$
\mathcal{S}_{0}=\int d^{4} x\left\{\frac{\mu^{2}}{2} V_{m}^{2}-\frac{1}{2 \times 4 !} F_{m n p q}^{2}-\frac{\mu}{6} \epsilon^{m n p q} V_{m}\left(H_{n p q}-C_{n p q}\right)\right\},
$$

where $H_{3}=d B_{2}$ is the field strength of a two-form field $B_{2}$, in form language. Field eqs. of $B$ gives

$$
d V=0 \quad \rightarrow V=-d \varphi .
$$

Plugging back in (2.10) one finds the original action (2.1). Alternatively, eliminating the vector field through its field eqs. leads to

$$
\begin{aligned}
& V^{m}=\frac{1}{6 \mu} \epsilon^{m n p q}\left(H_{n p q}-C_{n p q}\right), \\
& S_{\text {dual }}=\int d^{4} x\left\{-\frac{\mu^{2}}{12}\left(H_{n p q}-C_{n p q}\right)^{2}-\frac{1}{2 \times 4 !} F_{m n p q}^{2}\right\} .
\end{aligned}
$$

In the dual formulation, the massive three-form has one degree of freedom, matching the degree of freedom of the scalar in the original formulation. In the action (2.12) the three-form absorbed the two form $B_{2}$ and its axion in a generalization of the Stueckelberg mechanism (see for ex. $[45,46]$ ), which is transparent writing the action in the more compact form

$$
\mathcal{S}_{\text {dual }}=\int\left\{-\frac{\mu^{2}}{2}\left(d B_{2}-C_{3}\right) \wedge^{\star}\left(d B-C_{3}\right)-\frac{1}{2} F_{4} \wedge^{\star} F_{4}\right\} .
$$

\section{Three-form multiplet in supersymmetry and stabilizer multiplet in inflation}

The three-form multiplet in supersymmetry is defined as the real superfield [47-53]

$$
\begin{aligned}
U=\bar{U}= & B+i(\theta \chi-\bar{\theta} \bar{\chi})+\theta^{2} \bar{M}+\bar{\theta}^{2} M+\frac{1}{3} \theta \sigma^{m} \bar{\theta} \epsilon_{m n p q} C^{n p q} \\
& +\theta^{2} \bar{\theta}\left(\sqrt{2} \bar{\lambda}+\frac{1}{2} \bar{\sigma}^{m} \partial_{m} \chi\right)+\bar{\theta}^{2} \theta\left(\sqrt{2} \lambda-\frac{1}{2} \sigma^{m} \partial_{m} \bar{\chi}\right)+\theta^{2} \bar{\theta}^{2}\left(D-\frac{1}{4} \square B\right) .
\end{aligned}
$$

The difference between $U$ and a regular vector superfield $V$ is the replacement of the vector potential $V_{m}$ by the three-form $C^{n p q}$. In order to find correct kinetic terms, the analog 
of the chiral field strength superfield $W_{\alpha}$ for a vector multiplet is replaced by the chiral superfield [47]

$$
S=-\frac{1}{4} \bar{D}^{2} U \quad, \quad S\left(y^{m}, \theta\right)=M+\sqrt{2} \theta \lambda+\theta^{2}(D+i F),
$$

with $F$ defined as in (2.3). The definition (3.2) is invariant under the gauge transformation $U \rightarrow U-L$, where $L$ is a linear multiplet. Correspondingly, lagrangians expressed as a function of $S$ will have this gauge freedom. One can therefore choose a gauge in which $B=\chi=0$ in (3.1) and the physical fields are $M, \lambda$. The supersymmetrization of the coupling of the inflaton $\phi$ to the three-form is a superpotential mass term coupling a chiral superfield $\phi$ including the inflaton

$$
[\mu S \phi]_{F}+\text { h.c. }=[\mu(\phi+\bar{\phi}) U]_{D}-\mathcal{S}_{b}(\phi),
$$

where $\mathcal{S}_{b}(\phi)$ is a total derivative, given explicitly by

$$
\begin{aligned}
\mathcal{S}_{b}(\phi)=\mu \partial^{m}\left[\frac { 1 } { 4 } \left(B \partial_{m}(\phi\right.\right. & \left.+\bar{\phi})-\partial_{m} B(\phi+\bar{\phi})\right) \\
& \left.+\frac{1}{2 \sqrt{2}}\left(\chi \sigma_{m} \bar{\psi}+\psi \sigma_{m} \bar{\chi}\right)-\frac{i}{6} \epsilon_{m n p q}(\phi-\bar{\phi}) C^{n p q}\right] .
\end{aligned}
$$

The inflaton $\varphi$ is contained in the imaginary part of the lowest component $\phi_{1}=(\zeta+$ $i \varphi) / \sqrt{2}$. Notice that in the generalization of the stabilizer models proposed in $[14,15]$, the superpotential $W=f(\phi) S$ can also be re-written as a contribution to the Kahler potential

$$
[S f(\phi)]_{F}+\text { h.c. }=[(f(\phi)+\bar{f}(\bar{\phi})) U]_{D}-\mathcal{S}_{b}(f(\phi)),
$$

where $\mathcal{S}_{b}(f(\phi))$ is a boundary term generalizing (3.4) that we don't display here. However only for the linear case $f(\phi)=\mu \phi$ is the shift symmetry unbroken in the action, up to boundary terms. On the other hand, shift symmetry is preserved by additional terms in the Kahler potential of the type $[(\phi+\bar{\phi}) g(U)]_{D}$. However, only for a linear function $g(U)=U$ is this term invariant under gauge transformations of the three-form $U \rightarrow U-L$. The linear coupling $\mu[(\phi+\bar{\phi}) U]_{D}=[\mu \phi S]_{F}+$ h.c. + total deriv. is therefore uniquely singled out by requiring shift symmetry and three-form gauge symmetry.

Let us consider the simplest ${ }^{2}$ example of interest for applications to inflation, provided by the lagrangian containing the chiral superfields $S$ and $\phi$

$$
\begin{aligned}
K & =|S|^{2}+\frac{1}{2}(\phi+\bar{\phi})^{2}+\mathcal{S}_{b}, \\
W & =\mu S \phi
\end{aligned}
$$

where the boundary action $\mathcal{S}_{b}$ is given by

$$
\begin{aligned}
\mathcal{S}_{b} & =\mathcal{S}_{b}(\phi)+\mathcal{S}_{b}(C), \\
\mathcal{S}_{b}(\phi) & =\mu \int d^{4} \theta(\phi+\bar{\phi}) U-\mu\left(\int d^{2} \theta S \phi+\text { h.c. }\right), \\
\mathcal{S}_{b}(C) & =\frac{1}{2}\left[D^{\alpha}\left(S D_{\alpha} U-U D_{\alpha} S\right)+\text { h.c. }\right]
\end{aligned}
$$

\footnotetext{
${ }^{2}$ We will comment later on expected changes by considering a more general Kahler potential.
} 
and is needed, as in the previous section, in order to get consistent field eqs. The shift symmetric kinetic term $\frac{1}{2}(\phi+\bar{\phi})^{2}$ is equivalent in the global supersymmetry case, up to boundary terms which are innocent (unlike the ones containing the three-form), to the standard one $|\phi|^{2}$. We keep however this form for later generalizations to supergravity. The full lagrangian can also be written as only a contribution to the Kahler potential

$$
K=|S|^{2}+\frac{1}{2}(\phi+\bar{\phi})^{2}+\mu(\phi+\bar{\phi}) U+\mathcal{S}_{b}(C) .
$$

Notice that in the form (3.8) the coupling inflaton-three form is precisely in the form (2.5), which includes therefore the inflaton-dependent boundary term $\mathcal{S}_{b}(\phi)$, as seen from the explicit expression

$$
\begin{aligned}
{[(\phi+\bar{\phi}) U]_{D}=} & F_{\phi} M+\bar{F}_{\bar{\phi}} \bar{M}+D(\phi+\bar{\phi})-\frac{i}{6} \epsilon_{m n p q} \partial^{m}(\phi-\bar{\phi}) C^{n p q}-(\lambda \psi+\bar{\lambda} \bar{\psi}) \\
& +\partial^{m}\left[\frac{1}{4}\left(B \partial_{m}(\phi+\bar{\phi})-\partial_{m} B(\phi+\bar{\phi})\right)+\frac{1}{2 \sqrt{2}}\left(\chi \sigma_{m} \bar{\psi}+\psi \sigma_{m} \bar{\chi}\right)\right]
\end{aligned}
$$

Field equations determine the auxiliary fields to be

$$
\begin{aligned}
2 D+\mu(\phi+\bar{\phi}) & =0 \quad, \quad F=-\frac{i \mu}{2}(\phi-\bar{\phi})-f_{0}, \\
F_{\phi}+\mu \bar{S} & =0
\end{aligned}
$$

where $f_{0}$ is a flux allowed since $F$ is a field strength and not really an auxiliary field, such that its field eq. is $\partial_{n}\left(F+\frac{i \mu}{2}(\phi-\bar{\phi})\right)=0$. The final lagrangian is obtained after taking into account carefully the contribution of the boundary terms. The scalar potential is given by

$$
V=\mu^{2}|M|^{2}+\left|\mu \phi+i f_{0}\right|^{2}
$$

and display again the combination inflaton/flux similar to (2.8). As already discussed in the non-supersymmetric case and displayed in (2.9), the shift symmetry is broken to a discrete subgroup, with a corresponding change in the flux quantum.

Notice that for the purpose of finding the correct on-shell lagrangian and scalar potential, there is a simpler formulation in which $S$ is treated as a standard chiral superfield with $D+i F$ as standard auxiliary fields, no boundary terms are included, but the superpotential of the theory is changed according to [51-53]

$$
W(\phi, S) \rightarrow W^{\prime}(\phi, S)=W(\phi, S)+i f_{0} S
$$

which in our case becomes

$$
W^{\prime}=\mu S \phi+i f_{0} S .
$$

Similarly to (2.9), for quantized flux $f_{0}=n e^{2}$, there is a discrete remnant of the shift symmetry

$$
\phi \rightarrow \phi-\frac{i e^{2}}{\mu} \quad, \quad n \rightarrow n+1,
$$

interpreted in terms of membrane nucleation which induces the monodromy in the inflaton excursions. 


\subsection{Dual formulation}

The dual formulation starts from the master action

$$
K=|S|^{2}+\frac{\mu^{2}}{2} V^{2}+\mu^{2} V(U-L),
$$

where $V$ is a real vector superfield and $L$ is a linear multiplet satisfying $D^{2} L=\bar{D}^{2} L=0$, that can be expressed as a function of the unconstrained fermionic superfield $\Sigma_{\alpha}$ via $L=$ $D^{\alpha} \bar{D}^{2} \Sigma_{\alpha}+\bar{D}_{\dot{\alpha}} D^{2} \bar{\Sigma}^{\dot{\alpha}}$. Field eq. of the linear multiplet gives

$$
\mu V=\phi+\bar{\phi},
$$

which, after plugging back into (3.15), gives the original bulk Kahler potential (3.8). On the other hand, eliminating the vector superfield via its field eqs. leads to

$$
V=-U+L \quad, \quad K_{\text {dual }}=|S|^{2}-\frac{\mu^{2}}{2}(U-L)^{2} .
$$

The dual lagrangian (3.17) contains a massive three-form multiplet, which has precisely the same degrees of freedom as the original action containing two chiral superfields $S$ and $\phi$. Notice that the combination $U-L$ is the analog of the Stueckelberg combination $V-d \phi$ for a massive vector multiplet and is gauge invariant in the same sense. In the massive case, all bosonic $B, M, C_{m n p}$ and fermionic fields $\chi, \lambda$ are physical. The action (3.17) contains therefore four bosonic and four fermionic degrees of freedom, of mass $\mu$. They match of course the degrees of freedom of the chiral multiplet formulation in terms of the chiral fields $\phi, S$. Interestingly enough, in analogy with the non-supersymmetric starting point, the massive three-form multiplet contains both the inflaton and the stabilizer field, and its mass term drives chaotic inflation.

\subsection{Corrections to the inflaton potential}

Whereas the mass term (3.6) or equivalently the D-density $[(\phi+\bar{\phi}) U]_{D}$ is uniquely singled out by the shift symmetry and the three-form gauge symmetry, more general Kahler (or higher derivative) contributions can be considered. As shown in [39], corrections to the stabilizer Kahler potential, for ex. a term of the type $-\zeta(\bar{S} S)^{2}$ are actually needed in order to generate a large stabilizer mass during inflation, without changing the inflaton potential. A more general Kahler potential of the form $K(S, \bar{S}, \phi+\bar{\phi})$ does not change conceptually our discussion above provided it contains in its expansion the standard quadratic terms, and does not impact inflationary dynamics provided that its stabilizes the field $M$ to zero during inflation.

On the other hand, corrections to the inflaton potential arise from higher-derivative interactions. The simplest higher-derivative ghost-free correction to the effective action is of the form

$$
\frac{1}{\Lambda^{4}}\left[D^{\alpha} S D_{\alpha} S D_{\dot{\alpha}} \bar{S} D^{\dot{\alpha}} \bar{S}\right]_{D},
$$

where $\Lambda$ is an UV scale. This generates corrections of the type $\frac{1}{\Lambda^{4}} F^{4}$ to the effective action, of the type considered in the non-supersymmetric case in [7-9], which in this case lead to corrections to the inflaton potential $\delta V \sim \frac{\mu^{4} \varphi^{4}}{\Lambda^{4}}$. According to [7-9], such corrections to not affect significantly chaotic inflation provided that $\Lambda \gg M_{G U T}$. 


\section{Supergravity formulation of the three-form multiplet}

The supergravity embedding of the three-form multiplet was pioneered in [48-52, 54]. In what follows we use the notations and conventions of [55]. The chiral weight of the three-form multiplet $U$ in supergravity is zero, because it is real. The Weyl weight $w$, on the other hand, is arbitrary. It is convenient to take it equal to 2 , so in what follows $\left(c_{U}, w_{U}\right)=(0,2)$. We also define the chiral projector $\Sigma$, of weights $\left(c_{\Sigma}, w_{\Sigma}\right)=(3,1)$. In the old minimal supergravity, the compensator $S_{0}$ has weights $\left(c_{0}, w_{0}\right)=(1,1)$ and it is fixed at $S_{0}=\bar{S}_{0}=e^{K / 6}$ in order to define supergravity in the Einstein frame. All other chiral fields are defined in order to have zero chiral and Weyl weights. One can then define the analog of the chiral superfield $S$ in the previous section by ${ }^{3}$

$$
S=\frac{1}{S_{0}^{3}} \Sigma(U)=e^{-\frac{K}{2}} \Sigma(U) .
$$

The inflaton will be one of the matter fields with zero weights, such that an arbitrary supergravity lagrangian will be of the form

$$
\mathcal{S}=\left[-3 e^{-\frac{1}{3} K(\phi, S, \bar{\phi}, \bar{S})} S_{0} \bar{S}_{0}\right]_{D}+\left[S_{0}^{3} W(\phi, S)\right]_{F}
$$

Of particular interest in what follows for inflationary models is the mass-like term

$$
\left[\mu S_{0}^{3} \phi S\right]_{F}=[\mu(\phi+\bar{\phi}) U]_{D}-\mathcal{S}_{b}
$$

where $\mathcal{S}_{b}$ is a boundary term. In analogy with the rigid limit therefore, the would-be mass term does not break the shift symmetry. The boundary term is expected, similar to the rigid case, to be completely included in the lagrangian with the term $\mu(\phi+\bar{\phi}) U$ in the Kahler potential.

It was shown in $[51,52]$ that, similarly to the global supersymmetry case, the supergravity couplings of the three-form can be described by using the chiral superfield $S$, treated as a standard chiral superfield, with the modification (3.12) of the superpotential. This is the simplest approach that we will use in what follows.

\subsection{Chaotic inflation}

The lagrangian for chaotic inflation is provided by [10]

$$
\begin{aligned}
K & =\left|S^{2}\right|+\frac{1}{2}(\phi+\bar{\phi})^{2}, \\
W & =\mu S \phi+i f_{0} S,
\end{aligned}
$$

\footnotetext{
${ }^{3}$ Our definition (4.1) is the same as in Burgess et al. in [48-50] in the superconformal formalism and in Binétruy et al. in [48-52] in the Kahler superspace setup. These references were concerned with gaugino condensation in supersymmetry and supergravity and in this context the three-form multiplet was the Chern-Simons form of Yang-Mills theories, whereas in our case it is identified with the stabilizer multiplet in inflation. None of these references wrote explicitly boundary terms, which were discussed in the rigid case in [53]. Reference [54] contains a different, unrelated supergravity construction, containing a three-form as a compensator multiplet.
} 
where we added the flux contribution $f_{0}$ for practical calculations (allowing to compute naively scalar potential and field eqs.) and we neglected Kahler corrections for the stabilizer [39], which are important for the inflationary dynamics but not for our current discussion. We have shown in section 3 that in the global supersymmetry case there is a dual formulation in terms of a massive three-form multiplet, of lagrangian (3.17). The supergravity generalization is similar. Starting from the master action

$$
\mathcal{S}=\left[-3 e^{-\frac{1}{3} K(S, \bar{S}, \mu V)} S_{0} \bar{S}_{0}+\mu^{2} V(U-L)\right]_{D},
$$

where $V$ is a vector multiplet of weights $\left(c_{V}, w_{V}\right)=(0,0)$ and $L$ is a linear multiplet of the same weights as the three-form multiplet $U,\left(c_{L}, w_{L}\right)=(0,2)$, eqs. of motion of the linear multiplet and the action can be written as

$$
\begin{aligned}
\mu V & =\phi+\bar{\phi}, \\
\mathcal{S} & =\left[-3 e^{-\frac{1}{3} K(S, \bar{S}, \phi+\bar{\phi})} S_{0} \bar{S}_{0}+\mu(\phi+\bar{\phi}) U\right]_{D} .
\end{aligned}
$$

In the dual version, one uses the field eqs. of the vector multiplet

$$
\frac{\delta K}{\delta V} e^{-\frac{1}{3} K} S_{0} \bar{S}_{0}+\mu^{2}(U-L)=0 .
$$

For example for a quadratic form $K=\frac{\mu^{2}}{2} V^{2}$, after eliminating the vector multiplet $V=$ $-U+L$, we recover the mass term of a massive three-form multiplet (3.17).

\subsection{The Starobinsky model}

The equivalence between higher-derivative supergravity and standard supergravity with two chiral superfields was pioneered by Cecotti [35] and developed further in [36, 37]. In what follows, we discuss the Starobinsky model and duality in the case where one of the chiral fields contain the three form. In the simplest, chiral formulation, the Starobinsky model is given by

$$
\begin{aligned}
& K=-3 \ln \left(T+\bar{T}-\left|S^{2}\right|\right), \\
& W=\mu\left(T-\frac{1}{2}\right) S+i f_{0} S,
\end{aligned}
$$

where again $S$ is the chiral superfield containing the four-form field strength, but treated as a standard chiral superfield in (4.8), due to the addition of the flux superpotential linear term proportional to $f_{0}$. The inflaton $\varphi$ is defined here via the real part of $T$ :

$$
T=e^{\sqrt{\frac{2}{3} \varphi}}+i \sqrt{\frac{2}{3}} a,
$$

with $a$ being an axion. Similar to the previous cases, the model has a discrete shift symmetry acting on the axion field. If the flux is quantized $f_{0}=n e^{2}$, with $e$ being the elementary three-form electric charge and with our definition (4.9), the symmetry transformation is

$$
a \rightarrow a-\sqrt{\frac{3}{2}} \frac{e^{2}}{\mu} \quad, \quad n \rightarrow n+1
$$


but it does not involve the inflaton $\varphi$. This version of Starobinsky model cannot therefore be considered as "natural" in the sense that super-Planckian values of the inflaton cannot be reached by nucleating three-form membranes.

Dual gravitational formulation. The dual description starts from the lagrangian

$$
\begin{aligned}
\mathcal{S} & =-\left[\left(1+T+\bar{T}-|S|^{2}\right) S_{0} \bar{S}_{0}\right]_{D}+\left[\left(\mu T+i f_{0}\right) S S_{0}^{3}\right]_{F} \\
& =-\left[\left(1-|S|^{2}\right) S_{0} \bar{S}_{0}\right]_{D}+\left[T\left(\mu S-\frac{\mathcal{R}}{S_{0}}\right) S_{0}^{3}+i f_{0} S S_{0}^{3}\right]_{F},
\end{aligned}
$$

where in (4.11) $\mathcal{R}$ denotes the chiral gravity multiplet superfield and in the last equality we used the identity $[36,37,55]$

$$
\left[(f(\Lambda)+\bar{f}(\bar{\Lambda})) S_{0} \bar{S}_{0}\right]_{D}=\left[f(\Lambda) \mathcal{R} S_{0}^{2}\right]_{F}+\text { total derivative } .
$$

One can therefore eliminate the chiral multiplet $S$ in favor of the gravity multiplet $\mathcal{R}$, according to

$$
\mathcal{R}=\mu S_{0} S=\frac{\mu}{S_{0}^{2}} \Sigma(U)=\frac{1}{S_{0}} \Sigma\left(\bar{S}_{0}\right)
$$

where the last equality defines actually the chiral multiplet $\mathcal{R}$ in supergravity. In detail, the components of the chiral superfield $\mathcal{R}$ are

$$
\mathcal{R}=\left(\bar{u} \equiv S+i P, \gamma^{m n} \mathcal{D}_{m} \psi_{n},-\frac{1}{2} R-\frac{1}{3} A_{m}^{2}+i \mathcal{D}^{m} A_{m}-\frac{1}{3} u \bar{u}\right),
$$

where $u$ and $A_{m}$ are the "old minimal" auxiliary fields of $N=1$ supergravity and $\psi_{n}$ is the gravitino field. Notice that according to the prescription (3.12), if the flux term $f_{0}$ is included as in (4.11), $S$ can be treated as a standard chiral multiplet in the lagrangian. Let us however ignore this term and look at the duality with $S$ containing the four-form field strength. In components, (4.13) contains the duality relations

$$
\begin{aligned}
\bar{u} & =\mu M, & \gamma^{m n} \mathcal{D}_{m} \psi_{n} & =\sqrt{2} \mu \lambda, \\
-\frac{1}{2} R-\frac{1}{3} A_{m}^{2}-\frac{1}{3}\left|u^{2}\right| & =\mu D, & \mathcal{D}^{m} A_{m} & =\mu F .
\end{aligned}
$$

In the dual formulation, the vector multiplet auxiliary field $A_{m}$ of the old minimal supergravity is therefore replaced by the three-form $C_{m n p}$ and the duality relation (4.15) contain the duality $C_{3}={ }^{\star} A$, or in components $C_{m n p}=\epsilon_{m n p q} A^{q}$. Duality (4.13) also implies the relation $\mu(U-L)=S_{0} \bar{S}_{0}$. It is unclear to us if this could be interpreted as replacing the chiral compensator $S_{0}$ of the old minimal supergravity by a three-form compensator. ${ }^{4}$

For the dual lagrangian, the simplest option is probably to add the flux superpotential term $f_{0}$ as in (4.11) and treat $S$ as a standard chiral superfield. One therefore finds, in the old minimal supergravity formulation

$$
\mathcal{S}_{\text {dual }}=-\left[S_{0} \bar{S}_{0}-\frac{1}{\mu^{2}} \mathcal{R} \overline{\mathcal{R}}\right]_{D}+\left[i f_{0} \frac{\mathcal{R}}{\mu} S_{0}^{2}\right]_{F} .
$$

According to (4.12), the last, new term compared to the standard higher-derivative supergravity in (4.16), is a total derivative.

\footnotetext{
${ }^{4} \mathrm{~A}$ different formulation of supergravity with a three-form compensator multiplet was proposed some time ago in [54].
} 


\section{$5 \quad$ Embedding in string theory}

A natural interpretation of the inflaton in string theory is as a Wilson line [26-28, 32, 33], which being an internal component of a gauge field, it enjoys the shift symmetry as a remnant of the higher-dimensional gauge symmetry. On the other hand, the three-form origin could be one of the RR forms present in the closed string spectrum of type II strings. Let us give a suggestive example of one D5 brane in type IIB strings, without getting into various possible subtleties; it is by no means to be considered as a unique possibility. There is a $\mathrm{U}(1)$ gauge field living on the brane. In what follows we denote by $x_{5}, x_{6}$ the internal dimensions in the brane wordvolume and by $A_{5}, A_{6}$ the internal component of the gauge fields. After a suitable complexification (we take the complex structure of the torus $\tau=i$ to simplify the discussion)

$$
z=\frac{x_{5}+i x_{6}}{\sqrt{2}} \quad, \quad \phi=\frac{A_{6}+i A_{5}}{\sqrt{2}}
$$

the one-form gauge field and field strengths are

$$
A=A_{M} d x^{M}=A_{m} d x^{m}+2 \operatorname{Im}(\phi d z) \quad, \quad F=\frac{1}{2} F_{m n} d x^{m} \wedge d x^{n}+2 \operatorname{Im}(d \phi \wedge d z) .
$$

Type IIB strings have a four-form that can contain three-forms from the four-dimensional viewpoint

$$
C_{4} \supset C_{3}^{z} d z+\bar{C}_{3}^{z} d \bar{z}
$$

that in components read $C_{3}^{z}=\frac{1}{\sqrt{2} \times 3 !}\left(C_{m n p 5}-i C_{m n p 6}\right) d x^{m} \wedge d x^{n} \wedge d x^{p}$. Then the ChernSimons couplings of the RR forms to the brane worldvolume gauge field is given by

$$
q_{5} \int_{D 5} C \wedge e^{F} \supset \mu \int\left(d \phi \wedge \bar{C}_{3}^{z}+d \bar{\phi} \wedge C_{3}^{z}\right)=-\mu \int\left(\phi \wedge \bar{F}_{4}^{z}+\bar{\phi} \wedge F_{4}^{z}\right)+\text { total deriv. }
$$

where $q_{5}$ is the D5 brane RR charge and $\mu=q_{5} \int_{\mathcal{C}_{2}} \Phi \overline{\mathcal{C}}_{3}^{z}$, where $\mathcal{C}_{2}$ is the two-cycle wrapped by the brane and $\Phi, \overline{\mathcal{C}}_{3}^{z}$ are the internal profiles of the corresponding fields. The flux parameter $f_{0}$ of the previous sections is related by Hodge duality to the five-form flux along the internal space. One concrete setup is the orientifold of type IIB string by $\Omega^{\prime}=\Omega \mathcal{I}_{4}$, where $\mathcal{I}_{4}$ is the inversion of four (two complex) internal coordinates $z_{1}, z_{2}$. The RR 4 -form $C_{4}$ is odd under $\Omega$, but its components $C_{3}^{z_{1}}, C_{3}^{z_{2}} \sim \int_{\mathcal{C}_{1}} C_{4}$, integrals over one-cycles in the $z_{1}, z_{2}$ internal space are even. The D5 brane under consideration should wrap $z_{1}$ or $z_{2}$. In this case, the inflaton mass parameter $\mu$ is determined by the D5 brane charge $q_{5}$, and also by the wavefunction normalization of the Wilson line kinetic term, which depends in general on complex structure moduli. A small value $\mu \sim 10^{-5} M_{P}$ could then be obtained for extreme values of complex structure moduli.

Another possible realization is the type I string with magnetized [59-61] D9 branes. ${ }^{5}$ In this case, the Chern-Simons couplings of RR fields to D9 brane gauge fields are

$$
q_{9} \int_{D 9} C \wedge e^{F} \supset q_{9} \int_{D 9} C_{6} \wedge F \wedge F \supset q_{9} \int_{D 9} \epsilon^{m_{1} \cdots m_{4}} \epsilon^{i_{1} \cdots i_{6}} C_{m_{1} m_{2} m_{3} i_{1} i_{2} i_{3}} \partial_{m_{4}} A_{i_{4}}\left\langle F_{i_{5} i_{6}}\right\rangle,
$$

\footnotetext{
${ }^{5}$ In what follows $m_{1} \cdots m_{4}$ are spacetime indices, whereas $i_{1} \cdots i_{6}$ are internal indices.
} 
where $\left\langle F_{i_{5} i_{6}}\right\rangle$ is a magnetic flux and where $C_{6}$ is the RR six-form, dual to the RR two-form of type I string. Here the inflaton mass $\mu$ is given by an integral over the compact space of internal wavefunctions and the magnetic flux $\mu \sim q_{9} \int \mathcal{C} d \Phi\langle F\rangle$. The coupling (5.5) has a form similar to (5.4) with appropriate identification of fields. The flux parameter $f_{0}$ is here related to a seven form field strength flux or, by Hodge duality, by an internal three-form flux ${ }^{\star} F_{7}=F_{3}=f_{0} \Omega$, where $\Omega$ is the holomorphic $(3,0)$ form. Other string theory examples are discussed in [32, 33] (see also [62]). The importance of integrating out consistently four-dimensional three-forms in superstring compactifications was emphasized in [63].

Our discussion here only concerns the origin of the inflaton-stabilizer coupling. In a realistic string setup, other issues have to be addressed, like moduli stabilization and supersymmetry breaking (see for ex. [26-33]). They are however beyond the goals of this paper.

\section{Acknowledgments}

I would like to thank W. Buchmuller, S. Ferrara, R. Kallosh and J. Louis for very useful discussions and correspondence. This work has been supported in part by the ERC advanced grant "MassTeV" and the Alexander von Humboldt foundation. Special thanks are due to the Theory Group in DESY-Hamburg for hospitality during the whole project and to MITP-Mainz for hospitality during the last stages of the work.

Open Access. This article is distributed under the terms of the Creative Commons Attribution License (CC-BY 4.0), which permits any use, distribution and reproduction in any medium, provided the original author(s) and source are credited.

\section{References}

[1] A.H. Guth, The inflationary universe: a possible solution to the horizon and flatness problems, Phys. Rev. D 23 (1981) 347 [INSPIRE].

[2] A.D. Linde, Chaotic inflation, Phys. Lett. B 129 (1983) 177 [INSPIRE].

[3] A. Albrecht and P.J. Steinhardt, Cosmology for grand unified theories with radiatively induced symmetry breaking, Phys. Rev. Lett. 48 (1982) 1220 [INSPIRE].

[4] K. Freese, J.A. Frieman and A.V. Olinto, Natural inflation with pseudo-Nambu-Goldstone bosons, Phys. Rev. Lett. 65 (1990) 3233 [INSPIRE].

[5] J.E. Kim, H.P. Nilles and M. Peloso, Completing natural inflation, JCAP 01 (2005) 005 [hep-ph/0409138] [INSPIRE].

[6] R. Kappl, S. Krippendorf and H.P. Nilles, Aligned natural inflation: monodromies of two axions, Phys. Lett. B 737 (2014) 124 [arXiv:1404.7127] [INSPIRE].

[7] N. Kaloper and L. Sorbo, A natural framework for chaotic inflation, Phys. Rev. Lett. 102 (2009) 121301 [arXiv:0811.1989] [INSPIRE].

[8] N. Kaloper, A. Lawrence and L. Sorbo, An ignoble approach to large field inflation, JCAP 03 (2011) 023 [arXiv:1101.0026] [INSPIRE]. 
[9] N. Kaloper and A. Lawrence, Natural chaotic inflation and UV sensitivity, Phys. Rev. D 90 (2014) 023506 [arXiv: 1404.2912] [InSPIRE].

[10] M. Kawasaki, M. Yamaguchi and T. Yanagida, Natural chaotic inflation in supergravity, Phys. Rev. Lett. 85 (2000) 3572 [hep-ph/0004243] [INSPIRE].

[11] Planck collaboration, P.A.R. Ade et al., Planck 2013 results. XXII. Constraints on inflation, Astron. Astrophys. 571 (2014) A22 [arXiv:1303.5082] [INSPIRE].

[12] D.H. Lyth, What would we learn by detecting a gravitational wave signal in the cosmic microwave background anisotropy?, Phys. Rev. Lett. 78 (1997) 1861 [hep-ph/9606387] [INSPIRE].

[13] BICEP2 collaboration, P.A.R. Ade et al., Detection of B-mode polarization at degree angular scales by BICEP2, Phys. Rev. Lett. 112 (2014) 241101 [arXiv:1403.3985] [InSPIRE].

[14] R. Kallosh, A. Linde and T. Rube, General inflaton potentials in supergravity, Phys. Rev. D 83 (2011) 043507 [arXiv: 1011.5945] [InSPIRE].

[15] R. Kallosh, A. Linde, K.A. Olive and T. Rube, Chaotic inflation and supersymmetry breaking, Phys. Rev. D 84 (2011) 083519 [arXiv:1106.6025] [InSPIRE].

[16] K. Harigaya, M. Ibe, K. Schmitz and T.T. Yanagida, Dynamical chaotic inflation in the light of BICEP2, Phys. Lett. B 733 (2014) 283 [arXiv:1403.4536] [INSPIRE].

[17] K. Harigaya and T.T. Yanagida, Discovery of large scale tensor mode and chaotic inflation in supergravity, Phys. Lett. B 734 (2014) 13 [arXiv:1403.4729] [INSPIRE].

[18] S. Ferrara, A. Kehagias and A. Riotto, The imaginary Starobinsky model, Fortsch. Phys. 62 (2014) 573 [arXiv:1403.5531] [INSPIRE].

[19] J. Ellis, M.A.G. García, D.V. Nanopoulos and K.A. Olive, Resurrecting quadratic inflation in no-scale supergravity in light of BICEP2, JCAP 05 (2014) 037 [arXiv:1403.7518] [INSPIRE].

[20] T. Li, Z. Li and D.V. Nanopoulos, Chaotic inflation in no-scale supergravity with string inspired moduli stabilization, arXiv:1405.0197 [INSPIRE].

[21] R. Kallosh, A. Linde and A. Westphal, Chaotic inflation in supergravity after Planck and BICEP2, Phys. Rev. D 90 (2014) 023534 [arXiv:1405.0270] [INSPIRE].

[22] R. Kallosh, A. Linde and D. Roest, Large field inflation and double $\alpha$-attractors, JHEP 08 (2014) 052 [arXiv: 1405.3646] [INSPIRE].

[23] S.V. Ketov and T. Terada, Inflation in supergravity with a single chiral superfield, Phys. Lett. B 736 (2014) 272 [arXiv:1406.0252] [INSPIRE].

[24] W. Buchmüller, V. Domcke and K. Schmitz, The chaotic regime of D-term inflation, arXiv: 1406.6300 [INSPIRE].

[25] W. Buchmüller, E. Dudas, L. Heurtier and C. Wieck, Large-field inflation and supersymmetry breaking, JHEP 09 (2014) 053 [arXiv: 1407.0253] [INSPIRE].

[26] L.E. Ibáñez and I. Valenzuela, BICEP2, the Higgs mass and the SUSY-breaking scale, Phys. Lett. B 734 (2014) 354 [arXiv:1403.6081] [INSPIRE].

[27] A. Hebecker, S.C. Kraus and L.T. Witkowski, D7-brane chaotic inflation, Phys. Lett. B 737 (2014) 16 [arXiv:1404.3711] [INSPIRE].

[28] M. Arends et al., D7-brane moduli space in axion monodromy and fluxbrane inflation, Fortsch. Phys. 62 (2014) 647 [arXiv:1405.0283] [INSPIRE]. 
[29] E. Palti and T. Weigand, Towards large r from [p, q]-inflation, JHEP 04 (2014) 155 [arXiv: 1403.7507] [INSPIRE].

[30] R. Blumenhagen and E. Plauschinn, Towards universal axion inflation and reheating in string theory, Phys. Lett. B 736 (2014) 482 [arXiv:1404.3542] [INSPIRE].

[31] T.W. Grimm, Axion inflation in F-theory, Phys. Lett. B 739 (2014) 201 [arXiv:1404.4268] [INSPIRE].

[32] F. Marchesano, G. Shiu and A.M. Uranga, F-term axion monodromy inflation, JHEP 09 (2014) 184 [arXiv: 1404.3040] [INSPIRE].

[33] S. Franco, D. Galloni, A. Retolaza and A. Uranga, Axion monodromy inflation on warped throats, arXiv: 1405.7044 [INSPIRE].

[34] A.A. Starobinsky, A new type of isotropic cosmological models without singularity, Phys. Lett. B 91 (1980) 99 [INSPIRE].

[35] S. Cecotti, Higher derivative supergravity is equivalent to standard supergravity coupled to matter. 1, Phys. Lett. B 190 (1987) 86 [INSPIRE].

[36] S. Ferrara, R. Kallosh and A. Van Proeyen, On the supersymmetric completion of $R+R^{2}$ gravity and cosmology, JHEP 11 (2013) 134 [arXiv: 1309.4052] [INSPIRE].

[37] S. Cecotti and R. Kallosh, Cosmological attractor models and higher curvature supergravity, JHEP 05 (2014) 114 [arXiv: 1403.2932] [INSPIRE].

[38] S. Ferrara, R. Kallosh, A. Linde and M. Porrati, Minimal supergravity models of inflation, Phys. Rev. D 88 (2013) 085038 [arXiv:1307.7696] [INSPIRE].

[39] R. Kallosh and A. Linde, Superconformal generalizations of the Starobinsky model, JCAP 06 (2013) 028 [arXiv: 1306.3214] [INSPIRE].

[40] I. Antoniadis, E. Dudas, S. Ferrara and A. Sagnotti, The Volkov-Akulov-Starobinsky supergravity, Phys. Lett. B 733 (2014) 32 [arXiv:1403.3269] [INSPIRE].

[41] E. Silverstein and A. Westphal, Monodromy in the CMB: gravity waves and string inflation, Phys. Rev. D 78 (2008) 106003 [arXiv:0803.3085] [INSPIRE].

[42] L. McAllister, E. Silverstein and A. Westphal, Gravity waves and linear inflation from axion monodromy, Phys. Rev. D 82 (2010) 046003 [arXiv:0808.0706] [InSPIRE].

[43] L. McAllister, E. Silverstein, A. Westphal and T. Wrase, The powers of monodromy, JHEP 09 (2014) 123 [arXiv:1405.3652] [INSPIRE].

[44] R. Bousso and J. Polchinski, Quantization of four form fluxes and dynamical neutralization of the cosmological constant, JHEP 06 (2000) 006 [hep-th/0004134] [INSPIRE].

[45] F. Quevedo and C.A. Trugenberger, Phases of antisymmetric tensor field theories, Nucl. Phys. B 501 (1997) 143 [hep-th/9604196] [INSPIRE].

[46] M. Berasaluce-González, G. Ramírez and A.M. Uranga, Antisymmetric tensor $Z_{p}$ gauge symmetries in field theory and string theory, JHEP 01 (2014) 059 [arXiv:1310.5582] [INSPIRE].

[47] S.J. Gates Jr., Super p form gauge superfields, Nucl. Phys. B 184 (1981) 381 [INSPIRE].

[48] C.P. Burgess, J.-P. Derendinger, F. Quevedo and M. Quirós, Gaugino condensates and chiral linear duality: an effective Lagrangian analysis, Phys. Lett. B 348 (1995) 428 [hep-th/9501065] [INSPIRE]. 
[49] C.P. Burgess, J.P. Derendinger, F. Quevedo and M. Quirós, On gaugino condensation with field dependent gauge couplings, Annals Phys. 250 (1996) 193 [hep-th/9505171] [INSPIRE].

[50] P. Binetruy, M.K. Gaillard and T.R. Taylor, Dynamical supersymmetric breaking and the linear multiplet, Nucl. Phys. B 455 (1995) 97 [hep-th/9504143] [INSPIRE].

[51] P. Binetruy, F. Pillon, G. Girardi and R. Grimm, The three form multiplet in supergravity, Nucl. Phys. B 477 (1996) 175 [hep-th/9603181] [INSPIRE].

[52] P. Binetruy, G. Girardi and R. Grimm, Supergravity couplings: a geometric formulation, Phys. Rept. 343 (2001) 255 [hep-th/0005225] [INSPIRE].

[53] K. Groh, J. Louis and J. Sommerfeld, Duality and couplings of 3-form-multiplets in $N=1$ supersymmetry, JHEP 05 (2013) 001 [arXiv: 1212.4639] [INSPIRE].

[54] B.A. Ovrut and D. Waldram, Membranes and three form supergravity, Nucl. Phys. B 506 (1997) 236 [hep-th/9704045] [INSPIRE].

[55] D.Z. Freedman and A. Van Proeyen, Supergravity, Cambridge University Press, Cambridge U.K. (2012).

[56] R. Kallosh, L. Kofman, A.D. Linde and A. Van Proeyen, Superconformal symmetry, supergravity and cosmology, Class. Quant. Grav. 17 (2000) 4269 [Erratum ibid. 21 (2004) 5017] [hep-th/0006179] [INSPIRE].

[57] E. Cremmer, S. Ferrara, L. Girardello and A. Van Proeyen, Yang-Mills theories with local supersymmetry: lagrangian, transformation laws and super-Higgs effect, Nucl. Phys. B 212 (1983) 413 [InSPIRE].

[58] T. Kugo and S. Uehara, Improved superconformal gauge conditions in the $N=1$ supergravity Yang-Mills matter system, Nucl. Phys. B 222 (1983) 125 [INSPIRE].

[59] C. Bachas, A way to break supersymmetry, hep-th/9503030 [INSPIRE].

[60] R. Blumenhagen, L. Görlich, B. Körs and D. Lüst, Noncommutative compactifications of type-I strings on tori with magnetic background flux, JHEP 10 (2000) 006 [hep-th/0007024] [INSPIRE].

[61] C. Angelantonj, I. Antoniadis, E. Dudas and A. Sagnotti, Type I strings on magnetized orbifolds and brane transmutation, Phys. Lett. B 489 (2000) 223 [hep-th/0007090] [INSPIRE].

[62] B. Shlaer, Chaotic brane inflation, Phys. Rev. D 88 (2013) 103503 [arXiv:1211.4024] [INSPIRE].

[63] T.W. Grimm, T.-W. Ha, A. Klemm and D. Klevers, The D5-brane effective action and superpotential in $N=1$ compactifications, Nucl. Phys. B 816 (2009) 139 [arXiv:0811.2996] [INSPIRE]. 